\title{
Agents and Ambient Intelligence: Case Studies
}

Dante I. Tapia ${ }^{1}$, Ajith Abraham ${ }^{2}$, Juan M. Corchado ${ }^{1}$ and Ricardo S. Alonso ${ }^{1}$

${ }^{1}$ Departamento Informática y Automática. University of Salamanca

Plaza de la Merced s/n, 37008, Salamanca, Spain

\{dantetapia, corchado, ralorin\}@usal.es

${ }^{2}$ Machine Intelligence Research Labs (MIR Labs)

Scientific Network for Innovation and Research Excellence

P.O. Box 2259, Auburn, Washington 98071-2259, USA

ajith.abraham@ieee.org

\begin{abstract}
The significance that Ambient Intelligence (AmI) has acquired in recent years requires the development of innovative solutions. In this sense, the development of AmI-based systems requires the creation of increasingly complex and flexible applications. One of the most important aspects in Ambient Intelligence is the use of context-aware technologies in order to perceive stimuli from both the users and the environment. Thus, the information obtained must be managed by intelligent and self-adaptable technologies in order to provide an adequate interaction between the users and their environment. Agents and Multi-Agent Systems are one of these technologies. The agents have characteristics such as autonomy, reasoning, reactivity, social abilities and proactivity which make them appropriate for developing dynamic and distributed systems based on Ambient Intelligence, as they possess the capability of adapting themselves to the users and environmental characteristics. This paper presents several case studies where agents and contextaware technologies have been implemented to build AmI-based systems. These case studies expand the possibilities of Ambient Intelligence and get closer to its vision.
\end{abstract}

Keywords: Ambient Intelligence, Agents and Multi-Agent Systems, Distributed Architectures, Context-Aware Technologies

\section{Introduction}

People are currently surrounded by technology which tries to increase their quality of life and facilitate the daily activities. However, there are situations where technology is difficult to handle or people have a lack of knowledge to use it. Ambient Intelligence (AmI) tries to adapt the technology to the people's needs by means of omnipresent computing elements which communicate amongst them in a ubiquitous way (Lyytinen, et al. 2002). In addition, the continuous advancement in mobile computing makes it possible to obtain information about the context and also to react physically to it in more innovative ways (Jayaputera, et al., 2007). 
The context is defined as any information used to characterize the situation of an entity, which can be a person, a place or an object (Dey, et al., 2000). Thus, the context includes both the users and the environment information. This information is important for defining the interaction between users and the technology that surround them. For these reasons, it is necessary to continuously keep track of information about the users and their environment. The information may consist of many different parameters such as location, the building status (e.g. temperature or light), vital signs (e.g. heart rhythm or blood pressure), etc. Thus, most of the context information can be collected by distributed sensors throughout the environment and even the users themselves. Wireless Sensor Networks (WSNs) are used for gathering the information needed by Ambient Intelligence environments, whether in home automation, educational applications or healthcare. Some examples of possible WSN technologies are RFID, ZigBee or Bluetooth.

One key aspect in any AmI-based system is the use of context-aware technologies. Therefore, a correct information management is vital. It is not enough to gather information about the context, but that information must be processed by self-adaptable and dynamic mechanisms and methods that can react independently of each particular situation that arises. In this sense, agents and MultiAgent Systems (MAS) comprise one of the areas that can contribute expanding the possibilities of Ambient Intelligence. An agent can be defined as a computational system situated in an environment and is able to act autonomously in this environment to achieve its design goals (Wooldridge, 2002). Expanding this definition, we have that an agent is anything with the ability to perceive its environment through sensors and respond in the same environment through actuators, assuming that each agent may perceive its own actions and learn from the experience (Russell, et al., 1995). A multi-agent system is defined as any system composed of multiple autonomous agents with incomplete capabilities to solve a global problem, where there is no global control system, the data is decentralized and the computing is asynchronous (Wooldridge, 2002) (Jennings, et al., 1998). As can be seen, the definition itself of an agent and a multi-agent system is closely related to Ambient Intelligence.

The BISITE Research Group of the University of Salamanca has developed several systems based on Ambient Intelligence. This paper presents a brief resume of these developments in order to realize the potential of agents into Ambient Intelligence scenarios. The next section briefly describes some of the main concepts defined by Ambient Intelligence and explains why agents are feasible to be implemented in these kinds of developments. In addition, it is introduced several context-aware technologies that help the agents to obtain information from the users and their environment. Section 3 presents a brief description of some case studies developed by the BISITE Research Group: a system aimed at enhancing the assistance and healthcare for Alzheimer patients; a healthcare telemonitoring system that makes use of multiple context-aware technologies; a system aimed at improving security on industrial environments; and an architecture for developing distributed AmI-based Multi-Agent Systems. Finally, section 4 presents the conclusions of this paper.

\section{Why Agents in Ambient Intelligence?}

Ambient Intelligence (AmI) is an emerging multidisciplinary area based on ubiquitous computing (Weiser, 1993) which influences the design of protocols, communications, systems, devices, etc. Ambient Intelligence proposes new ways of interaction between people and technology, making it suited to the needs of individuals and the environment that surrounds those (Aarts, et al., 2003). Ambient Intelligence tries to adapt the technology to the people's needs by proposing three basic concepts: ubiquitous computing, ubiquitous communication and intelligent user interfaces. Ambient Intelligence offers a great potential to improve quality of life and simplify the use of technology by offering a wider range of personalized services and providing users with easier and more efficient ways to communicate and interact with other people and systems. However, the development of systems that clearly fulfill the needs of AmI is difficult and not always satisfactory. It requires a joint development of models, techniques and technologies based on dynamic services.

An AmI-based system consists of a set of human actors and adaptive mechanisms which work together in a distributed way. Those mechanisms provide on demand personalized services and stimulate users through their environment according specific situation characteristics. As can be 
seen in Figure 1, the basic life cycle of an AmI-based system begins by obtaining information about the users and their environment by means of a monitoring service. The information is gathered through sensors or people. Then, the information is analyzed and processed by an information manager which gives consistency to data and store it. Once the information is processed, there are defined and personalized the activities to be executed and also the users that will be affected. The decisions are sent to the actors (i.e. people or devices) to execute the corresponding actions and stimulate both the users and their environment. The user can change its role during the process and trigger events that influence the context (i.e. users and their environment).

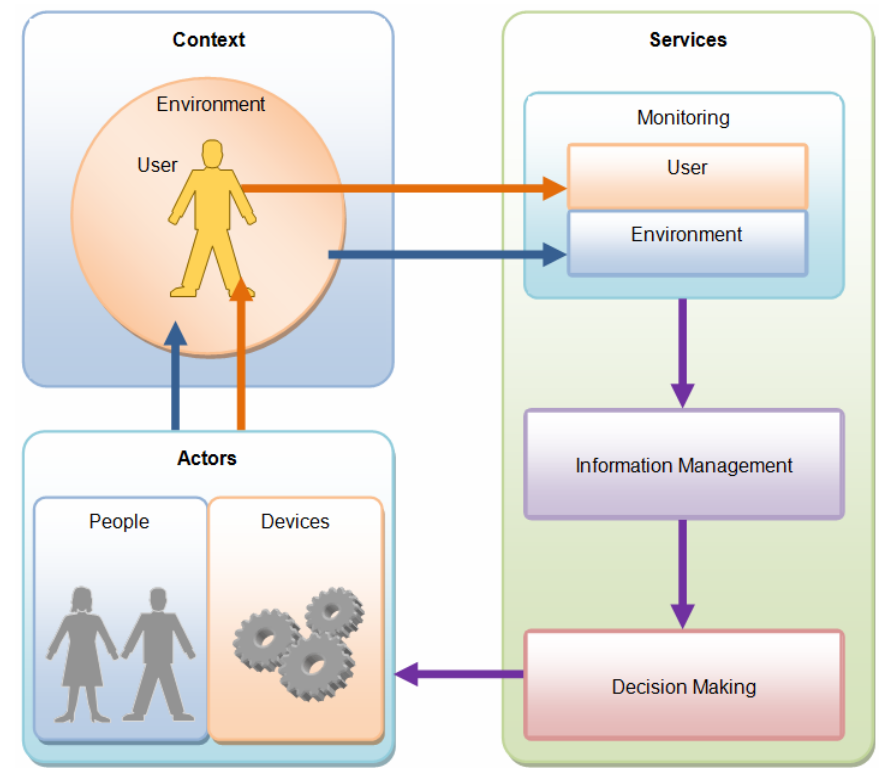

Fig. 1. Life cycle of an AmI-based system

The vision of AmI assumes seamless, unobtrusive, and often invisible but controllable interactions between humans and technology. AmI provides new possibilities for solving a wide scope of problems. It also proposes a new way to interact between people and technology, where this last one is adapted to individuals and their context. Ambient Intelligence shows a vision where people are surrounded by intelligent interfaces merged in daily life objects, creating computingcapable environments with intelligent communication and processing, serving people by means of a simple, natural and effortless human-system interaction.

The emergence of Ambient Intelligence involves substantial changes in the design of functional architectures, since it is necessary to provide features which enable a ubiquitous computing and communication and also an intelligent interaction with users. One of the most prevalent alternatives in distributed architectures is agent and multi-agent systems. There are several agent frameworks and platforms (Martin, et al., 1999) (Sycara, et al., 2003) (Bellifemine, et al., 1999) which provide a wide range of tools for developing distributed multi-agent systems. The development of agents is an essential piece in the analysis of data from distributed sensors and gives those sensors the ability to work together and analyze complex situations, thus achieving high levels of interaction with humans (Pecora, et al., 2007). Agent and multi-agent systems have been successfully applied to several Ambient Intelligence scenarios, such as education, culture, entertainment, medicine, robotics, etc. (Tapia, et al., 2006) (Sadeh, et al., 2005) (Schön, et al., 2005). Furthermore, agents can use reasoning mechanisms and methods in order to learn from past experiences and to adapt their behaviour according the context (Corchado, et al., 2008).

In the next section, it is described four AmI-based case studies developed by the BISITE Research Group of the University of Salamanca, Spain. These developments implement agents and wireless sensor in order to obtain stimuli from the context and react autonomously according each situation. 


\section{Case Studies}

This section presents several case studies where Ambient Intelligence has been successfully applied: A multi-agent system aimed at enhancing the assistance and healthcare for Alzheimer patients; a telemonitoring system aimed at enhancing remote healthcare of dependent people at their homes; a multi-agent system for managing and monitoring surveillance routes for security guards on industrial environments; and an architecture which facilitates the integration of multiagent systems, distributed services and applications to optimize the construction of Ambient Intelligence environments.

As stated in previous sections, a key aspect for the construction of Ambient Intelligence systems is obtaining context information through sensors. With the advances of electronic and computer technologies, most of the information can be collected by means of distributed sensors throughout the environment and even the people themselves. All the developments presented in this section share several technologies that allow the agents to obtain information about the context. One of these technologies is the Radio Frequency IDentification (RFID), a wireless communication technology used to identify and receive information about humans, animals and objects on the move. An RFID system contains basically four components: tags, readers, antennas and software. Mainly used in industrial/manufacturing, transportation and distribution, there are other growing sectors, including healthcare (ITAA, 2004). Another technology is ZigBee, a low cost, low power consumption, two-way, wireless communication standard developed by the ZigBee Alliance (ZigBee, 2006). ZigBee is designed to be embedded in consumer electronics, home and building automation, industrial controls, PC peripherals, medical sensor applications, toys and games, and is intended for home, building and industrial automation purposes, addressing the needs of monitoring, control and sensory network applications (ZigBee, 2006). Finally, the Bluetooth standard allows implementing multiple WPAN (Wireless Personal Area Network) or WBAN (Wireless Body Area Network) applications, including biomedical sensors that make possible to obtain useful context information from the state of the human users. The information collected through all these technologies is processed by the agents, providing self-adaptive capabilities to changes in the environment and user necessities.

\subsection{Alzheimer Healthcare}

ALZ-MAS (ALZheimer Multi-Agent System) (Corchado, et al., 2008) is a multi-agent system aimed at enhancing the assistance and healthcare for Alzheimer patients. The main functionalities in the system include reasoning and planning mechanisms (Glez-Bedia and Corchado, 2002) that are embedded into the agents, and the use of several context-aware technologies to acquire information from users and their environment. 


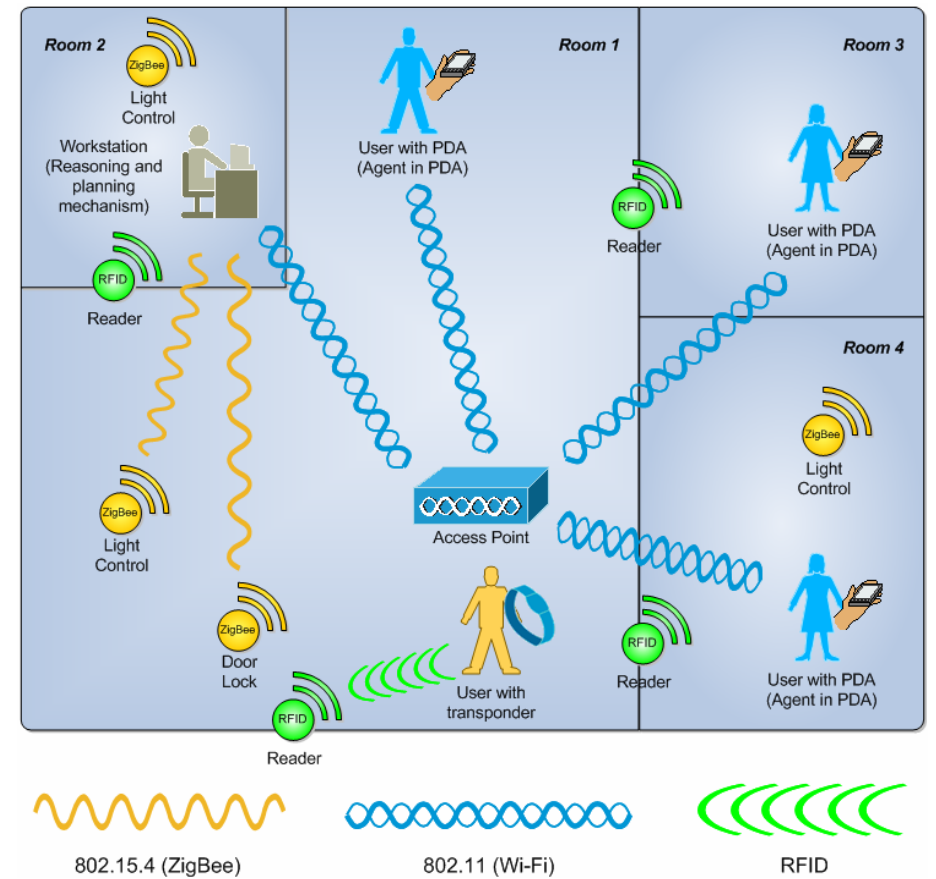

Fig. 2. ALZ-MAS basic schema

The ALZ-MAS structure has five different agents, each one with specific roles and capabilities: User Agent, SuperUser Agent, ScheduleUser Agent, Admin Agent and Devices Agents. As can be seen on Figure 2, ALZ-MAS makes use of RFID, Wi-Fi networks and ZigBee devices, providing the agents with automatic and real time information about the context. These technologies also allow the users interacting with their surroundings, controlling and managing physical services (i.e. heating, lights, switches, etc.). All the information is processed by the agents, specially the Devices Agent which runs on a Workstation. The Devices Agent monitors the users' location (continuously obtaining/updating data from RFID readers), interacts with the ZigBee devices to receive information and control physical services, and also checks the status of the Wi-Fi devices connected to the system (e.g. PDA). Figure 3 shows a RFID reader, a couple of RFID wrist tags and a ZigBee development board used in ALZ-MAS. The information obtained is sent to the Admin Agent to be processed. All hardware is someway integrated to agents, providing automatic and real time information about the environment that is processed by the agents to automate tasks and manage multiple services.

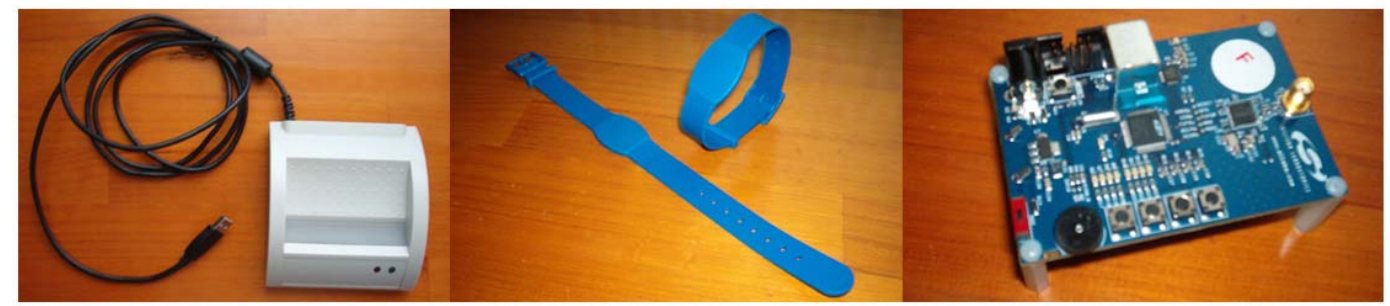

Fig. 3. A RFID reader (left), RFID wrist tags (center) and ZigBee development board (right) used in ALZ-MAS

ALZ-MAS has demonstrated that it can improve the security and healthcare efficiency through monitoring and automating medical staff's work and patients' activities, facilitating working shifts organization and reducing time spent on routine tasks. RFID, Wi-Fi and ZigBee technologies supply the agents with valuable information about the environment, contributing to a ubiquitous, non-invasive, high level interaction amongst users, system and the environment. 


\subsection{Telemonitoring Homecare}

A telemonitoring system aimed at enhancing remote healthcare of dependent people at their homes has also been developed (Alonso, et al., 2009). The main contribution of this development is the use of an experimental architecture (Tapia, et al., 2009a) that allows the interconnection of heterogeneous Wireless Sensor Networks (i.e. multiple technologies) and is based on the Ambient Intelligence paradigm. This architecture formalizes the integration of services, communications and wireless technologies to automatically obtain information from users and the environment in an evenly distributed way, focusing on the characteristics of ubiquity, awareness, intelligence and mobility.

As shown on Figure 4, the system infrastructure consists of a network of ZigBee devices designed to cover the home of each patient to be monitored. There is a ZigBee remote control carried by the monitored patient that includes a button which can be pressed in case of remote assistance or urgent help. Moreover, there are several ZigBee sensors that obtain information about the environment in which the user lives and that physically response to the changes. There are also several Bluetooth biomedical sensors placed over the patient's body. Biomedical sensors allow the system to acquire continuously data about the vital signs of the patient. All ZigBee and Bluetooth devices can both offer and invoke functionalities (i.e. services) in the network. There is also a computer connected to a remote healthcare telemonitoring center via Internet for forwarding the possible alerts to the caregivers and allowing them to communicate with patients.

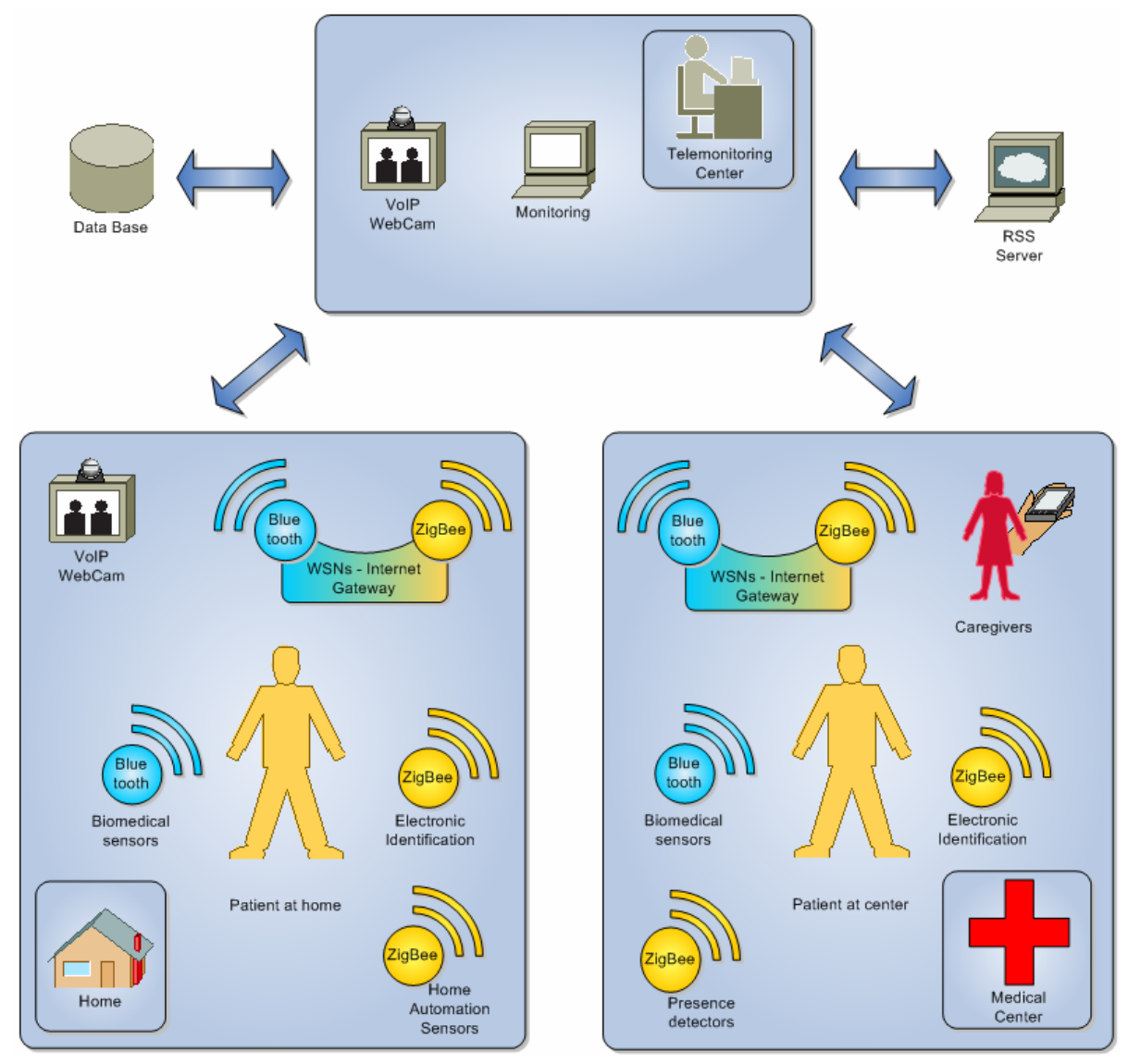

Fig. 4. Telemonitoring system basic schema

The system is mainly focused on monitoring tasks. However, it provides useful facilities to the patients and caregivers. For example, the remote center can consult RSS (Really Simple Syndication) sources from external and internal web servers in order to obtain weather reports or entertainment options for patients and inform them of their scheduled medical staff visits. Moreover, the system is not only intended for teleassistance but also for home automation. Furthermore, each patient is not only monitored at its home, but also at its medical center when he or she arrives to it for an appointment with its doctor. The ZigBee remote control carried by each 
patient has a unique electronic label that identifies him or her. There are ZigBee and Bluetooth networks throughout the medical center so that the patient's ZigBee identification label and its Bluetooth biomedical sensors can automatically connect to them.

From the AmI point of view, the most significant aspect of this development is the use of wireless devices from different technologies acting as a single network. The use of these technologies provides a high level of interaction with the users, which is an essential factor in constructing AmI environments. This kind of interaction is achieved by means of technology that is embedded, non-invasive and transparent for users and developers.

\subsection{Optimization of Surveillance Routes}

We have also developed a multi-agent system for managing and monitoring surveillance routes for security guards on industrial environments (Tapia, et al., 2009b). The system obtains automatic and real-time information about the context to schedule and monitor the security guards activities. The users interact with the system in a simple, natural and intuitive way, using a set of wireless technologies and software agents with reasoning and planning mechanisms. This system allows scheduling and distribution of surveillance routes amongst available security guards. The routes assigned are automatically and real-time monitored to ensure the accomplishment of the security guards working shifts. The system interacts with users through a set of mobile devices (PDAs) and wireless communication technologies (Wi-Fi, GPRS and RFID). These technologies and devices work in a distributed way, providing the users a flexible and easy access to the system services.

As shown on figure 5, the surveillance routes system is composed of five different kinds of agents: Guard Agent, Manager Agent, Planner Agent, Controller Agent and Advisor Agent. The agents in the system calculate the surveillance routes depending on the security guards available, the working shifts and the distance to be covered in the facilities. A supervisor (person) can set the possible routes, defining the areas that must be supervised, which can be modified according the scenario or changes in the environment. The system has the ability to re-plan the routes automatically considering the security guards available. It is also possible to track the workers activities (routes completion) over the Internet.

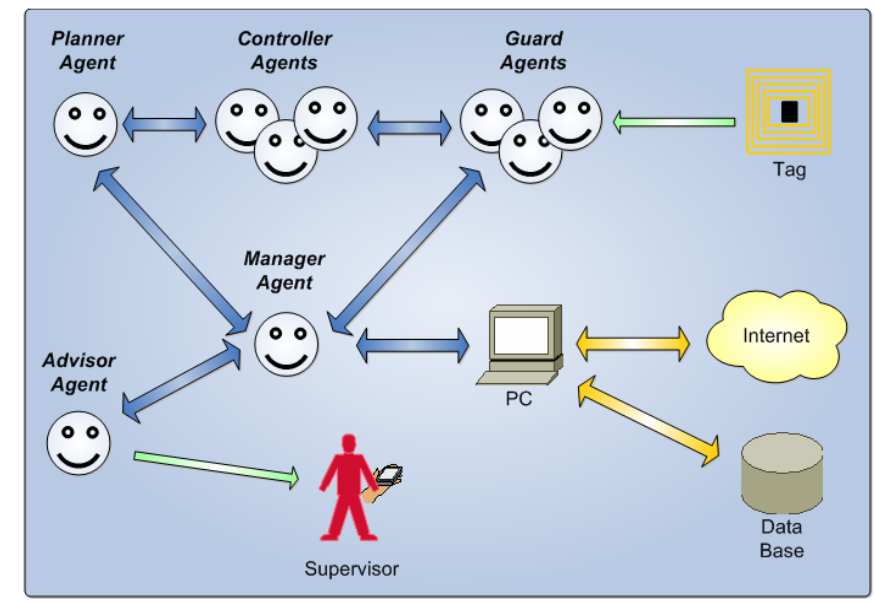

Fig. 5. Surveillance routes system basic schema

RFID is a key technology in this development. The RFID configuration for this system consists of a mesh of tags distributed all over the building. Each tag, named control point is related to an area which must be covered by the security guards. Each security guard carries on a PDA with a RFID reader to register the completion of each control point. The information is sent via wireless to a central computer to be processed. The use of wireless technologies, such as Wi-Fi, RFID, or GPRS provides an adequate communication infrastructure that the agents can use to obtain information about the context. With this information, the system can adapt services and interact with users according a specific situation in an easy, natural and ubiquitous way to solve some of daily life problems. This way, the system can be easily adapted to other scenarios with similar characteristics, providing a simple but powerful tool to optimize human resources and monitor the staff activities. 


\subsection{A Multi-Agent Architecture for Building Ambient Intelligence Systems}

FUSION@ (Tapia, et al., 2008) is a novel architecture which integrates a Service-Oriented Architectures (SOA) (Cerami, 2002) approach with intelligent agents for building systems based on the Ambient Intelligence paradigm. The architecture focuses on distributing the majority of the systems' functionalities into remote and local services and applications. It also proposes a new and easier method of building distributed multi-agent systems, where the functionalities of the systems are not integrated into the structure of the agents; rather they are modeled as distributed services and applications which are invoked by the agents acting as controllers and coordinators. This model provides a flexible distribution of resources and facilitates the inclusion of new functionalities in highly dynamic environments. One of the advantages of FUSION@ is that the users can access the system through distributed applications, which run on different types of devices and interfaces (e.g. computers, cell phones or PDAs).

Figure 6 shows the basic schema of FUSION@. This architecture proposes a new perspective, where multi-agent systems and SOA-based services are integrated to provide ubiquitous computation, ubiquitous communication and intelligent interfaces facilities. The FUSION@ framework defines four basic blocks: Applications, Services, Agents Platform and Communication Protocol. This framework has been designed following the SOA model, but adding the applications block which represents a fundamental part in Ambient Intelligence: the interaction with users. These blocks provide all the functionalities of the architecture. There are pre-defined agents that provide the basic functionalities of the architecture: CommApp Agent, CommServ Agent, Directory Agent, Supervisor Agent, Security Agent, Admin Agent and Interface Agent. Interface Agents were designed to be embedded in users' applications and are simple enough to allow them to be executed on mobile devices, such as cell phones or PDAs. FUSION@ exploits the agents' characteristics to provide a robust, flexible, modular and adaptable solution that can cover most requirements of a wide diversity of Ambient Intelligence projects.

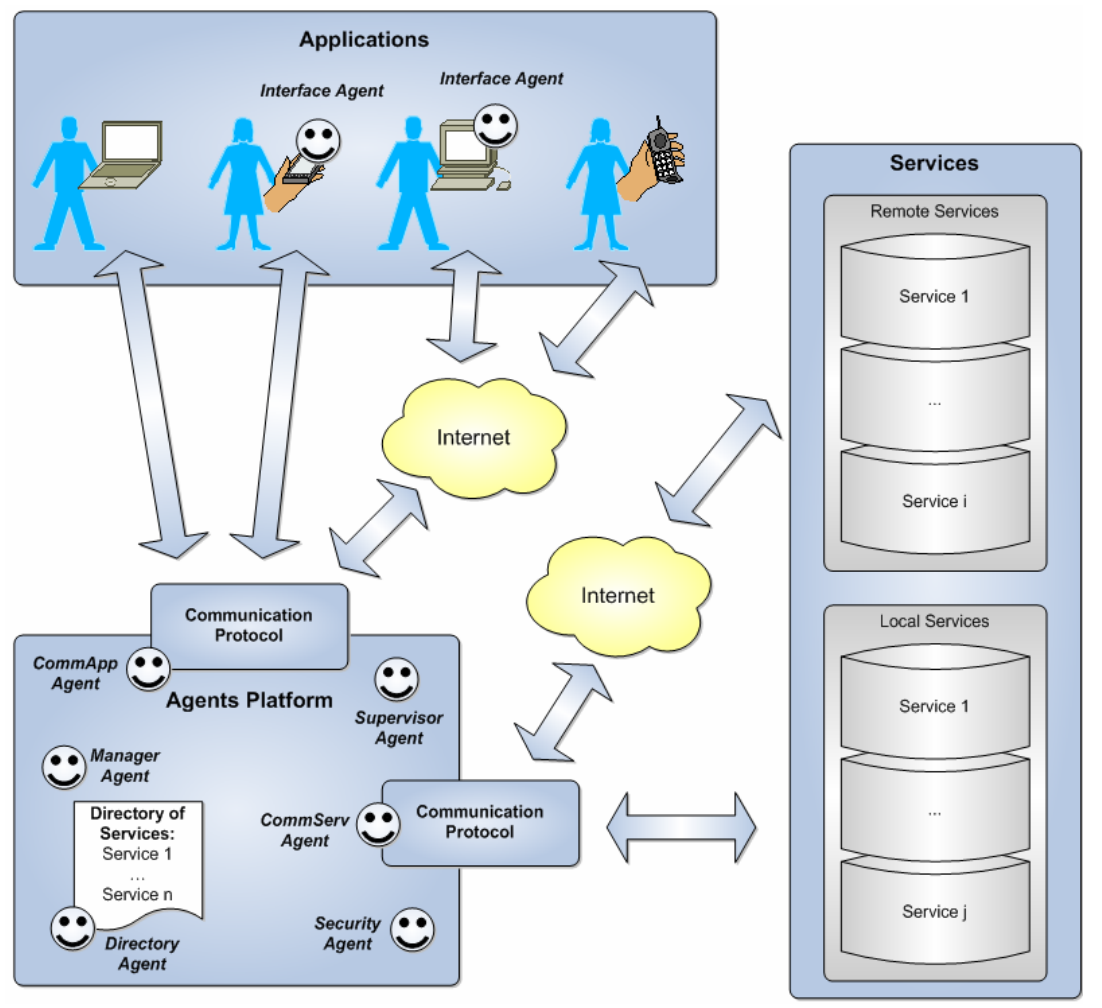

Fig.6. FUSION@basic schema

FUSION@ was mainly designed to develop systems based on Ambient Intelligence. Furthermore, FUSION@ has been employed to develop an improved version of ALZ-MAS, described in section 3.1. 


\section{Conclusions}

Ambient Intelligence is an emerging multidisciplinary area based on ubiquitous computing that proposes new ways of interaction between people and technology, adapting them to the needs of users and their environment. Ambient Intelligence improves the quality of life of the people providing them with easier and more efficient ways to communicate and interact with other people and systems.

Ambient Intelligence based applications involve complex design and implementation stages. This is because such applications must be highly distributed, dynamic and scalable, as well as ubiquitous for users from both software and hardware point of views. One of the most successful approaches to Ambient Intelligence is agents and multi-agent systems.

In a multi-agent system, each agent represents an entity with reasoning and social abilities and a high level of interaction with other systems and users, including reactive and proactive behaviors. These characteristics give the agents several advantages versus other approaches when implementing Ambient Intelligence applications, since the interaction of agents with users and the environment can be modeled by means of human-like behaviors.

This paper has presented four case studies developed by the BISITE Research Group of the University of Salamanca in order to demonstrate the suitability of agents and multi-agent systems when implementing Ambient Intelligence. These developments provide a ubiquitous, pervasive, high level interaction between the users and their environment. Future work includes the improvement of most of case studies presented, as well as the development of new multi-agent systems. In addition, we are currently integrating agents with other distributed paradigms as Service Oriented Architectures, as well as Wireless Sensor Networks technologies (e.g. ZigBee and Bluetooth) to gather information about the context in a more efficient way.

Acknowledgments. This work has been supported by the Spanish Ministry of Science and Technology project TIN2006-14630-C03-03.

\section{References}

Aamodt, A., \& Plaza, E. (1994). Case-Based Reasoning: foundational Issues, Methodological Variations, and System Approaches. AI Communications, 7 (1), 39-59.

Aarts, E., \& Roovers, R. (2003a). Embedded system design issues in ambient intelligence. In T. Basten, M. Geilen, \& H. d. Groot, Ambient intelligence: Impact on Embedded System Design (pp. 11-29). Norwell, MA: Kluwer Academic Publishers.

Alonso, R. S., García, Ó., Saavedra, A., Tapia, D. I., de Paz, J. F., \& Corchado, J. M. (2009). Heterogeneous Wireless Sensor Networks in a Telemonitoring System for Homecare. In Proceedings of IWANN (2) 2009, 663-670.

Cerami, E. (2002). Web Services Essentials Distributed Applications with XML-RPC, SOAP, UDDI \& WSDL (1st ed.). O'Reilly \& Associates, Inc.

Corchado, J. M., Bajo, J., De Paz, Y., \& Tapia, D. I. (2008). Intelligent Environment for Monitoring Alzheimer Patients, Agent Technology for Health Care. Decision Support Systems, 
382-396.

Dey, A. K., \& Abowd, G. D. (2000). Towards a Better Understanding of Context and ContextAwareness. In Proceedings of the CHI 2000 Workshop on The What, Who, Where, When, and How of Context-Awareness. The Hague, Netherlands.

Bellifemine, F., Poggi, A., \& Rimassa, G. (1999). Jade: A FIPA-compliant agent framework. In Proceedings of the Fourth International Conference on the Practical Application of Intelligent Agents and Multi-Agent Technology (PAAM’99), 97-108.

González-Bedia, M., \& Corchado, J. M. (2002). A planning Strategy based on Variational Calculus for Deliberative Agents. Computing and Information Systems Journal, 10, 2-14.

ITAA. (2004). Radio Frequency Identification. RFID...coming of age. Information Technology Association of America.

Jayaputera, G. T., Zaslavsky, A. B., \& Loke, S. W. (2007). Enabling run-time composition and support for heterogeneous pervasive multi-agent systems. Journal of Systems and Software , 80 (12), 2039-2062.

Jennings, N. R., Sycara, K., \& Wooldridge, M. (1998). A Roadmap of Agent Research and Development. (N. R. Jennings, K. Sycara, \& M. Georgeff, Eds.) Autonomous Agents and Multi-Agent Systems Journal , 1 (1), 7-38.

Lyytinen, K., \& Yoo, Y. (2002). Issues and Challenges in Ubiquitous Computing. Communications of the ACM , 45 (12), 63-65.

Martin, D., Cheyer, A., \& Moran, D. (1999). The open agent architecture: a framework for building distributed software systems. Applied Artificial Intelligence , 13 (1/2), 91-128.

Pecora, F., \& Cesta, A. (2007). Dcop for smart homes: A case study. Computational Intelligence, 23 (4), 395-419.

Russell, S., \& Norvig, P. (1995). Artificial Intelligence: A modern approach. Englewood Cliffs, NJ, USA: Prentice-Hall Series in Artificial Intelligence.

Sadeh, N., Gandon, F., \& Kwon, O. B. (2005). Ambient Intelligence: The MyCampus Experience. Carnegie Mellon University.

Schön, B., O'Hare, G. M., Duffy, B. R., Martin, A. N., \& Bradley, J. F. (2005). Agent Assistance for 3D World Navigation. Lecture Notes in Computer Science, 3661, 499-499.

Sycara, K., Paolucci, M., Van Velsen, M., \& Giampapa, J. (2003). The RETSINA MAS Infrastructure. Autonomous Agents and Multi-Agent Systems, 7 (1/2), 29-48. 
Tapia, D. I., Bajo, J., De Paz, J. F., \& Corchado, J. M. (2006). Hybrid Multi-Agent System for Alzheimer Health Care. In R. Solange Oliveira, \& A. C. Roque da Silva Filho (Ed.), In Proceedings of HAIS 2006. Ribeirao Preto, Brasil.

Tapia, D. I., Rodríguez, S., Bajo, J. \& Corchado, J. M. (2008). FUSION@, A SOA-Based Multiagent Architecture. In Proceedings of DCAI 2008, 99-107.

Tapia, D. I., Alonso, R. S., de Paz, J. F., \& Corchado, J. M. (2009a): Introducing a Distributed Architecture for Heterogeneous Wireless Sensor Networks. In Proceedings of IWANN (2) 2009, 116-123.

Tapia, D. I., De Paz, J. F., Rodríguez, S., \& Corchado, J. M. (2009b). Sistema multiagente para la gestión y monitorización de rutas de vigilancia. IEEE Latin America Transactions, 6 (3), In Press.

Weiser, M. (1993). Some computer science issues in ubiquitous computing. Communications of the ACM. Special issue on computer augmented environments: back to the real world, 36 (7), 75-84.

Wooldridge, M. (2002). An Introduction to MultiAgent Systems. Chichester, England: John Wiley \& Sons.

ZigBee. (2006). ZigBee Specification Document 053474r13. ZigBee Standards Organization. ZigBee Alliance. 